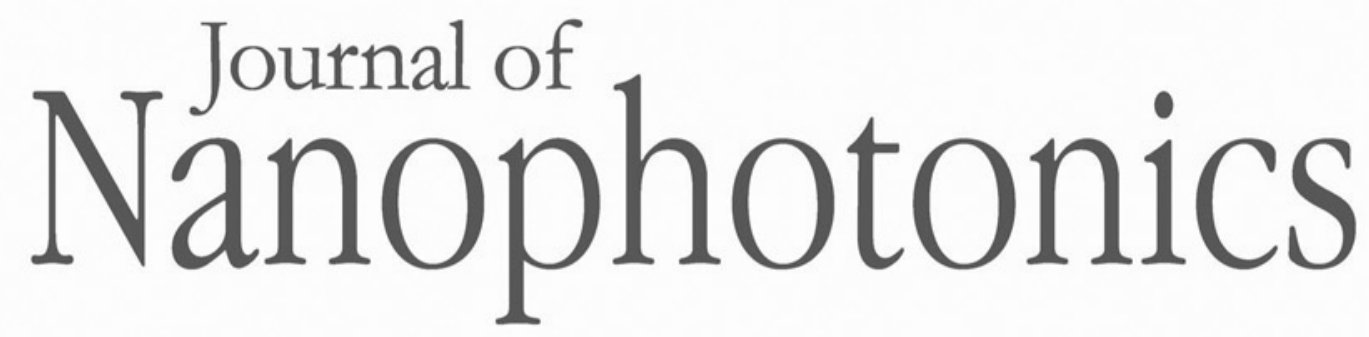

Nanophotonics.SPIEDigitalLibrary.org

\title{
Graphene-based wideband metamaterial absorber for solar cells application
}

\author{
Patrick Rufangura
}

Cumali Sabah 


\title{
Graphene-based wideband metamaterial absorber for solar cells application
}

\author{
Patrick Rufangura ${ }^{a}$ and Cumali Sabah ${ }^{b, c}$ 网 \\ ${ }^{a}$ Middle East Technical University-Northern Cyprus Campus (METU-NCC), Sustainable \\ Environment and Energy Systems (SEES), Kalkanli, Guzelyurt, TRNC/Mersin 10, Turkey \\ ${ }^{b}$ Middle East Technical University-Northern Cyprus Campus (METU-NCC), Department \\ of Electrical and Electronics Engineering, Kalkanli, Guzelyurt, TRNC/Mersin 10, Turkey \\ ${ }^{c}$ Middle East Technical University—Northern Cyprus Campus (METU-NCC), Kalkanli \\ Technology Valley (KALTEV), Kalkanli, Guzelyurt, TRNC/Mersin 10, Turkey
}

\begin{abstract}
A wideband metamaterial (MTM) absorber based on a concentric ring resonator is discussed at visible frequencies. The proposed structure offers a wideband absorption response, where absorption of $>70 \%$ is gained for the frequency ranging from 537.91 to $635.73 \mathrm{THz}$. The analysis is conducted on the components of the proposed structure to understand the origin of wideband absorption. Furthermore, a graphene monolayer sheet is integrated to the proposed MTM absorber to optimize its absorptivity, where the studies show enhancement of the absorptivity of the proposed structure up to $26 \%$ from its initial absorptivity. MTM absorbers of this kind have potential applications in solar cells. (C) 2017 Society of Photo-Optical Instrumentation Engineers (SPIE) [DOI: 10.1117/1.JNP11.036008
\end{abstract}

Keywords: metamaterial; absorber; wideband; graphene; solar cells.

Paper 17078 received May 25, 2017; accepted for publication Jul. 10, 2017; published online Aug. 1, 2017.

\section{Introduction}

Over the past few decades, metamaterials (MTM) have greatly attracted the attention of many researchers due to their simultaneous negative electric permittivity and magnetic permeability, which are unavailable in nature. MTM, which are made of periodic arrangements of the subwavelength unit-cell structures, are manmade composite materials. ${ }^{\text {. }}$ Through carefully designing the geometry of an MTM unit-cell structure, some interesting electromagnetic (EM) responses can be attained, such as a negative index of refraction, reverse Doppler effects, backward wave propagation,, $\mathrm{B}^{\mathrm{B}}$ and so on. Several applications have been invented due to MTM and its exotic

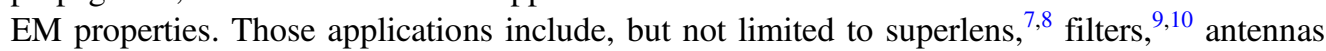

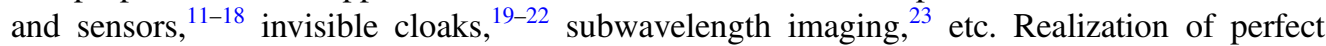
absorber (PA) devices is one of the greatest applications of MTM. The well-tailored MTM can be made to produce a media with zero (or very negligible) transmission and reflection of incident EM radiation, resulting in thorough absorption of all incident radiations at a certain range of frequencies and polarization angles. 270 MTM-based absorbers have demonstrated the ability to produce high (near perfect) absorptivity, which enabled the creation of absorber devices, including high absorber solar cells. 1 Normally, a unit cell of an MTM consists of a metallic layer, a dielectric substrate, and a ground plane. A perfect absorption can be realized by tuning $\varepsilon(\omega)$ and $\mu(\omega)$ separately so that the impedance of the MTM is matched to the freespace's impedance. Absorption can further be optimized by employing a ground metal plane of thickness greater than its skin depth ${ }^{3}$ in the frequencies of interest. The first MTM perfect absorber (MTMA) was demonstrated by Landy et al. ${ }^{-4}$ in 2008; a unit cell of their MTMA structure consists of a metallic split ring and a cut wire sandwiched with a dielectric substrate. Since then, MTM-based PAs have gained significant interest and different MTMAs have been

*Address all correspondence to: Cumali Sabah, E-mail: abah@metuledutt

$1934-2608 / 2017 / \$ 25.00$ (C) 2017 SPIE 
proposed from radio frequency to optical frequency ranges. band, multiband, and broadband MTM absorber structures have been proposed in some articles. important responses, such as polarization independency and wide angles of incidence, ${ }^{5}$ there is still a problem in the wideband MTMA designs that display perfect absorption for the high frequencies range, which limits their potential applications in solar cells. On the other hand, adding a graphene monolayer sheet on the MTMA unit-cell structure causes enhancement of their absorption coefficient and bandwidth. ${ }^{\mathrm{Q}}$ Interesting electronic transport properties far better than those of metals and semiconductors, high conductivity, and the excellent mechanical properties of graphene made it a good candidate in the MTM industry, where graphene sheets are used for MTM absorber designs in order to manipulate the propagating path of EM waves, which leads to optimization of absorption characteristics of MTM absorber structures

In this work, a wideband MTMA structure with nearly perfect absorption in the visible frequency region of the solar spectrum is proposed and numerically characterized; the proposed MTMA design consists of circular concentric ring resonators (CCRRs). A complete analysis concerning graphene's ability to increase the absorptions of the MTMA is provided, and it was concluded that addition of a graphene monolayer sheet in the proposed MTMA structure optimizes the wideband absorptivity character of the proposed MTM.

\section{Design, Simulation, and Discussion}

To design a reliable and highly efficient MTMA requires minimization of the reflection and transmission of incident radiations, manipulating the complex interactions of the MTM with the EM wave ${ }^{2}$ Generally, the absorption is described as $A(\omega)=1-R(\omega)-T(\omega)$, where $A(\omega), R(\omega)$, and $T(\omega)$ represent frequency $(\omega)$ dependent absorptivity, reflectivity, and transmission rate, respectively. It is obvious that if one wants to efficiently optimize the absorber, transmission and reflectivity need to be minimized to the very minimum possible coefficient. One method to tackle reflectivity is to adjust the MTMA effective impedance $Z_{\text {eff }}(\omega)$ so as to match it to the free-space impedance $Z_{0}$. In this work, the $T(\omega) \approx 0$ for a metallic ground plane whose thickness is larger than the skin depth is used. Thus, the total absorptivity of the proposed MTMA was calculated as $A(\omega)=1-R(\omega)$. The values of $R(\omega)$ and $T(\omega)$ can be extracted from the square of the frequency-dependent $S$-parameters $S_{11}(\omega)$ and $S_{12}(\omega)$.

In this study, a structure that was reported in our previous work ${ }^{\mathrm{G}}$ is utilized, then further study was extended to this work for integration of graphene on the same structure to understand graphene's effects on the absorptivity of the MTMA. The proposed MTMA is provided in Fig. 1. A unit cell of the structure consists of three circular ring resonators and each resonator is responsible for generating EM resonance once coupled with the dielectric space and ground metallic plane. Thus, three resonances were produced at three different resonant frequencies. All metallic layers were designated to be gold ( $\mathrm{Au}$ ) whose electric conductivity is $4.561 \times 10^{7} \frac{\mathrm{S}}{\mathrm{m}}$. $\mathrm{Au}$ is chosen based on its well-known properties such as ability to stand against extreme heat and high reflectance in the presence of high frequency EM radiations. The intermediate dielectric layer is gallium arsenide (GaAs) lossy with electric permittivity and loss tangent delta of 12.94 and 0.006, respectively. GaAs is preferred mainly due to its outstanding electron mobility and saturated electron velocity. In addition, GaAs is insensitive to excessive heat due to its wide bandgap and has a direct bandgap, which makes it an outstanding material for fabrication of devices for absorption and emission of light. The properties of glass Pyrex are electric permittivity and lossy tangent delta of 4.82 and 0.0054 , respectively. Glass Pyrex is selected for its outstanding transitivity of visible frequencies and its ability to stand against heat expansion.

The geometry parameters of the suggested MTMA unit cell are $a=17 \mathrm{~nm}, e=102 \mathrm{~nm}$, $g=146.2 \mathrm{~nm}, d=57.8 \mathrm{~nm}, c=27.2 \mathrm{~nm}, d=40.8 \mathrm{~nm}, t=103.4 \mathrm{~nm}, z=103.4 \mathrm{~nm}, x=$ $530 \mathrm{~nm}, n$ (thickness of glass Pyrex) $=16 \mathrm{~nm}$, and $k$ (CCRRs' thickness) $=6.8 \mathrm{~nm}$.

The numerical simulations for the proposed MTMA unit cell are performed with a full-wave EM simulator, which employs a finite integration technique (FIT) 6 6 The MTMA unit cell is intended to work in the visible frequency ranging from 450 to $800 \mathrm{THz}$. The incident EM radiations polarize in a way that the $E$-field and $H$-field propagate in parallel with the structure plane, 

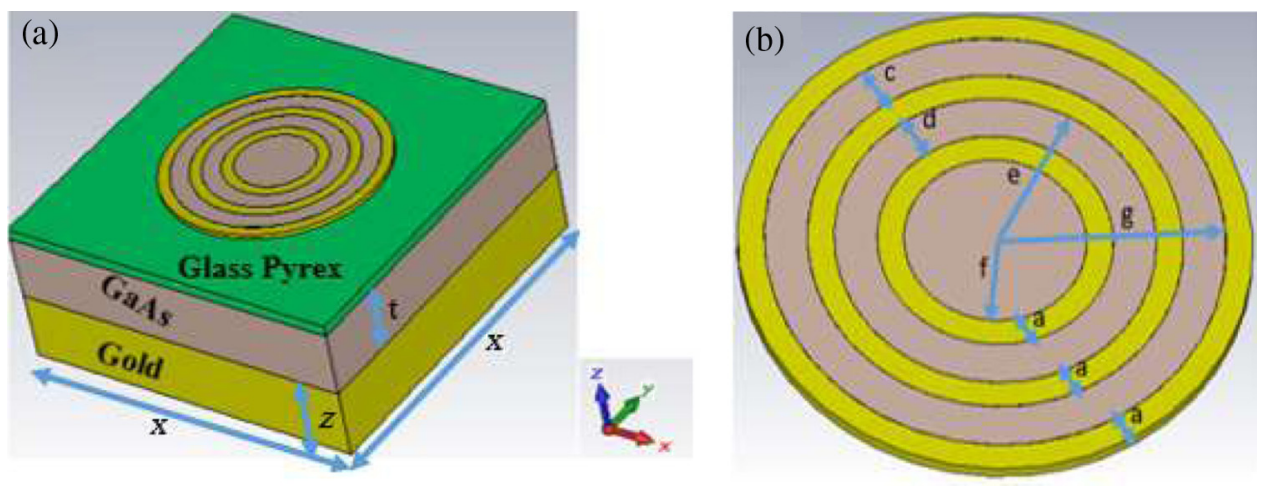

Fig. 1 (a) The proposed MTMA unit cell and (b) the top CCRRs with their geometric labels.

while wave vector $(k)$ is perpendicular to the geometric plane of the unit cell structure. The boundary conditions are set to periodic boundaries along $X$ and $Y$ directions, while the open and add space boundaries are selected for the simulations environment ( $Z$-plane).

The numerical simulation results for the proposed MTMA unit cell are reported in Fig. 目. As can be seen in Fig. 2(a), a wideband is gained at the resonant frequency, which believed to be a result of enhanced three-band resonant absorptions that join together to produce a wideband with a perfect absorption of $99.99 \%$. This is understood to result from independent EM resonances shaped by the strong coupling of each one of the three ring resonators with the dielectric substrate and with the ground metallic plane.

To comprehend the source of the observed wideband absorption characteristics for the MTMA unit cell in this study, numerical analysis is conducted for each resonator. In Fig. 2(b),
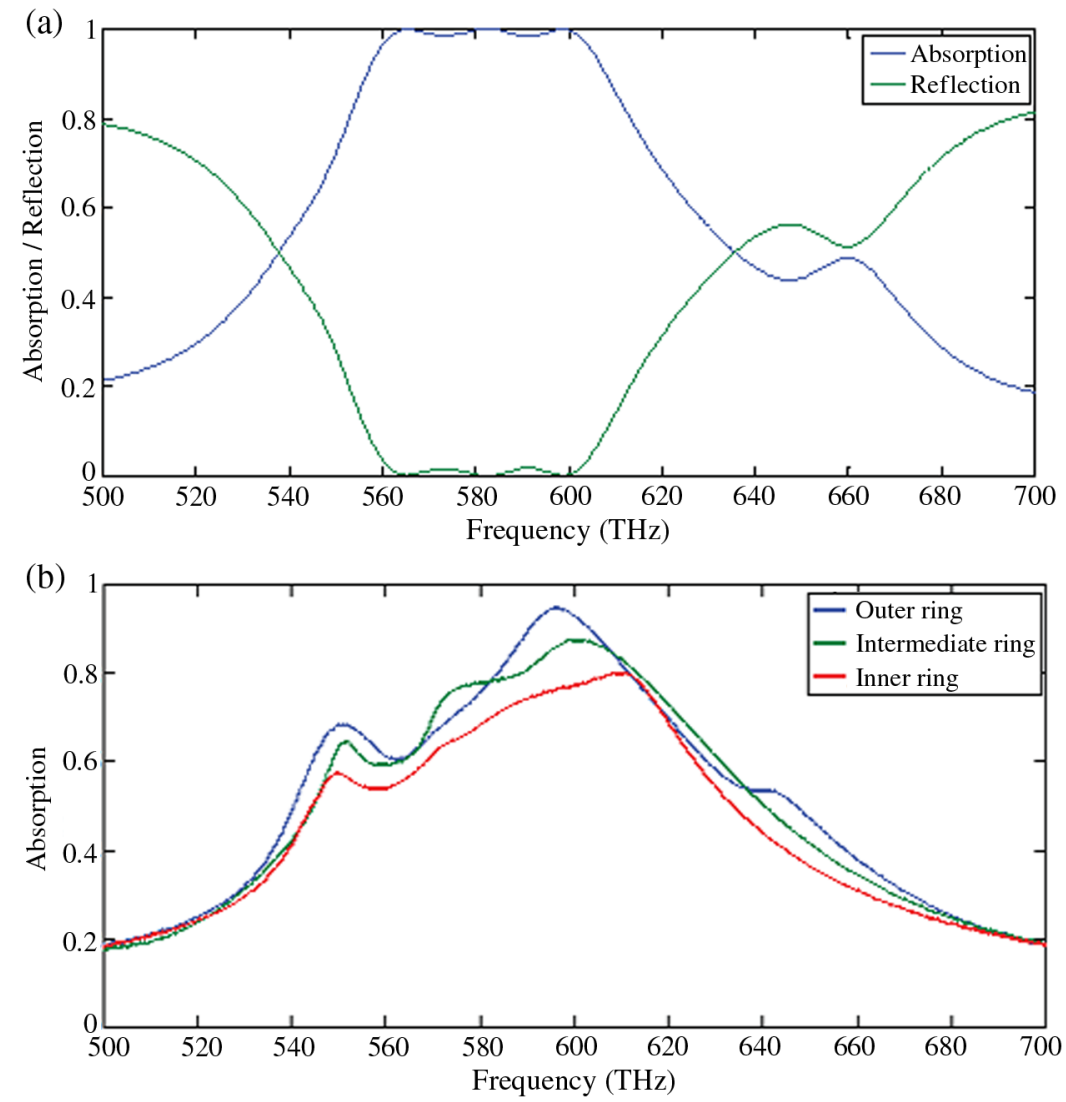

Fig. 2 (a) Simulated absorption and reflection rate for the proposed wideband MTMA unit cell and (b) simulated absorption rate of each ring resonator. 
the simulated absorption for each resonator is shown. One can notice that each resonator accounts for a single-band absorption resonance of about $90 \%$ absorption, while a combination of the three resonators generates strong electric and magnetic field resonances. This results in the enhancement of the total absorption gained by a unit cell and also produces a wideband absorption response.

\section{Integration of Graphene Monolayer Sheet on the Proposed MTMA Unit Cell}

In order to optimize the absorptivity of the proposed structure, a graphene monolayer sheet is integrated with it, where a layer of glass Pyrex lossy on the top of a dielectric spacer is substituted by the graphene sheet. In addition, a graphene layer is used to shield the top face of the resonators' surface. The simulation of graphene is made possible by using its surface conductivity, which can be controlled by chemical potential or bias electrostatic voltage and regulating graphene transport properties. The permittivity of graphene is calculated using Eq. (11):

$$
\varepsilon_{\mathrm{g}}=\frac{\sigma_{\mathrm{g}}}{(\mathrm{n} \omega)}+\varepsilon_{0}
$$

where $\varepsilon_{\mathrm{g}}, \varepsilon_{0}$, and $\left(\sigma_{\mathrm{g}}\right)$ symbolize the permittivity of graphene and free-space and the conductivity of graphene, respectively, while $n$ and $\omega$ represent the thickness of the graphene sheet and working frequency, respectively.

The Kubo-equation [Eq. (2)], which is generally used for modelling of conductivity $\left(\sigma_{\mathrm{g}}\right)$ of graphene, 906 is applied in this study and the obtained values are used in Eq. (1) to determine the permittivity of graphene:
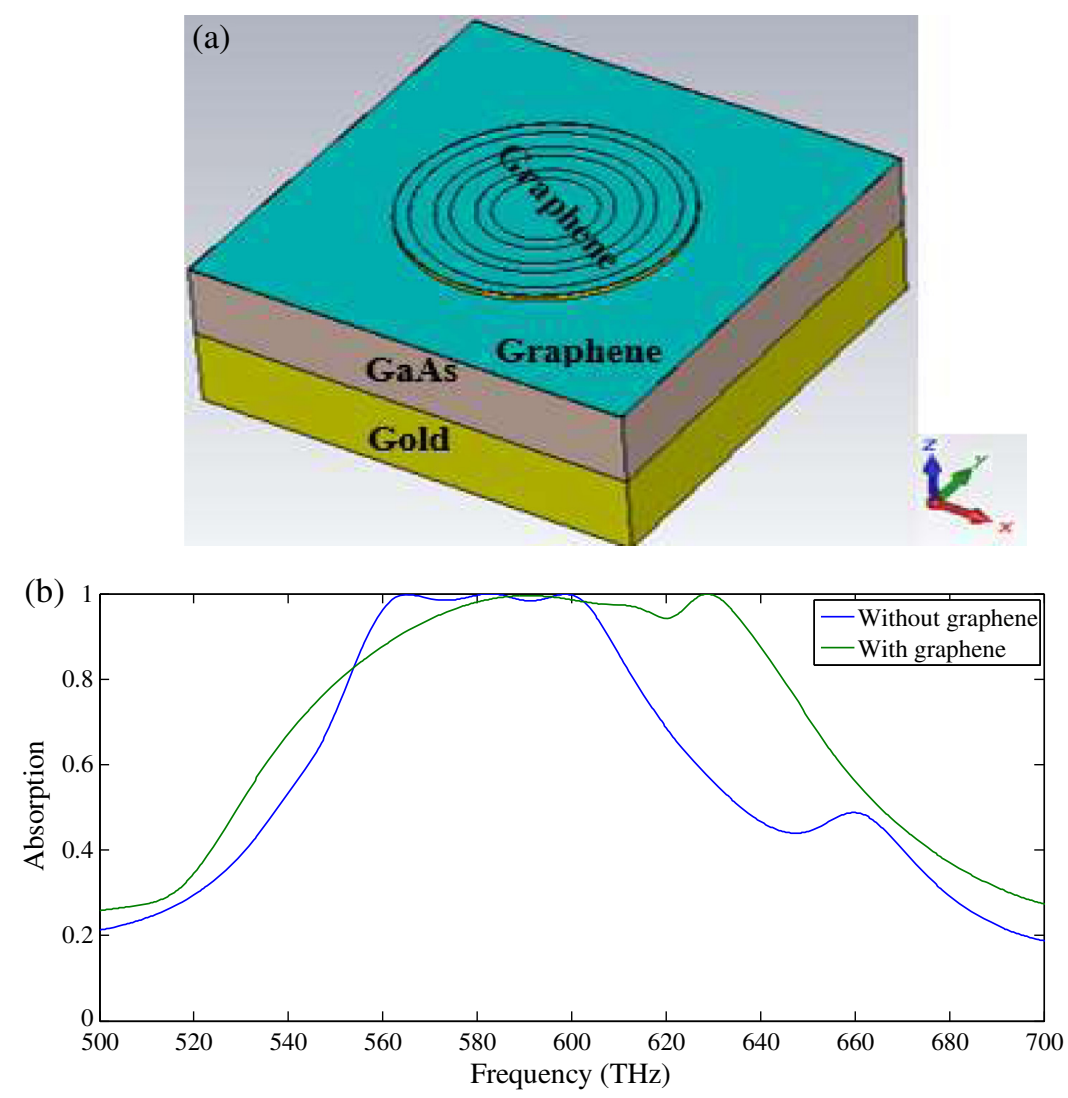

Fig. 3 (a) The proposed wideband MTMA structure with use of graphene monolayer sheet and (b) the simulated absorption of the proposed design with and without using graphene monolayer sheet. 

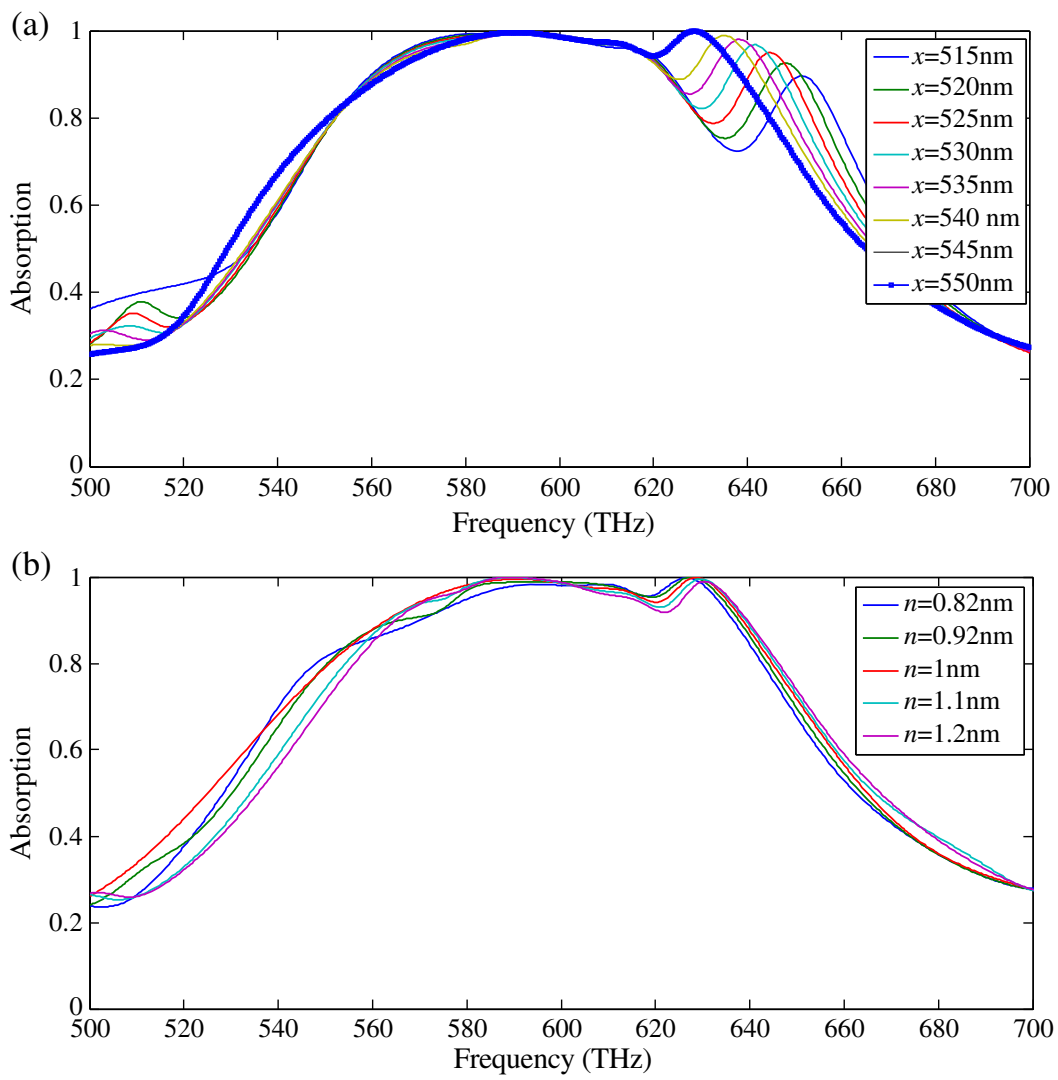

Fig. 4 Absorption characteristics of the proposed graphene-based wideband MPA structure under different geometric parameters. (a) Different value of graphene periodicity " $x$ " and (b) different thickness " $n$ " of graphene sheet.

$$
\sigma_{\mathrm{g}}=\frac{i e^{2}}{4 \pi \hbar} \ln \left[\frac{2\left|\mu_{c}\right|-(\omega+i 2 \Gamma) \hbar}{2\left|\mu_{c}\right|+(\omega+i 2 \Gamma) \hbar}\right]+\frac{i e^{2}}{\pi \hbar^{2}(\omega+i 2 \Gamma)}\left[\frac{\mu_{\mathrm{c}}}{k_{\mathrm{B}} T}+2 \ln \left(e^{-\mu_{c} / k_{\mathrm{B}} T}+1\right)\right],
$$

where $e, k_{\mathrm{B}}$, and $\hbar$ are universal constants, which symbolize the charge of an electron $\left(1.6 \times 10^{-19} \mathrm{C}\right)$, Boltzmann's constant $\left(1.3806 \times 10^{-23} \mathrm{~m}^{2} \mathrm{~kg} \mathrm{~s}^{-2} \mathrm{~K}^{-1}\right)$, and Planck's constant $\left(1.0545 \times 10^{-34} \mathrm{~m}^{2} \mathrm{~kg} \mathrm{~s}^{-1}\right)$, respectively. $T$ characterizes temperature and is taken to be room temperature $(300 \mathrm{~K})$ in this study; $\mu_{\mathrm{c}}$ and $\Gamma$ denote physical parameters of the graphene monolayer sheet and are responsible for chemical potential (Fermi energy) and intrinsic losses/ phonological scattering rate, in order. $\Gamma=\frac{\hbar}{\tau}$, and $\tau$ denotes electron relaxation time.

The input value for the phonological scattering rate is fixed to $\Gamma=1 \times 10^{-2} \mathrm{eV}$ in reference to the estimated maximum value of the electron mobility in graphene ${ }^{-}$In this study, the operational frequency $\omega$ is the visible spectrum range ranging from 450 to $800 \mathrm{THz}$ and the thickness ( $n$ ) of graphene is taken to be $1 \mathrm{~nm}$. By using the model in Eq. (2), conductivity was calculated at different chemical potentials $\left(\mu_{\mathrm{c}}\right)$ (bias electrostatic voltage) and by using (1) the permittivity of graphene was determined. The calculated permittivity is integrated in the computer simulator, which uses FIT in order to numerically analyze the contribution of graphene on the absorption characteristics of the proposed wideband MTMA structure. In Fig. B, enhanced wideband absorption results for the proposed structure are reported.

It can seen from Fig. B that an improved absorption bandwidth for the proposed structure is gained, which is a result of replacing the glass Pyrex layer with a graphene monolayer sheet and also by wrapping the resonators' face with the sheet of graphene, which causes excitation of surface plasmonic waves and leads to enhancement of the absorption bandwidth of the MTMA. Adding a graphene sheet on the proposed MTMA structure unit cell increases its absorption bandwidth up to $26 \%$ of its initial bandwidth, where an absorption rate of more than $90 \%$ covers a wide frequency range of $137 \mathrm{THz}$. 
The geometrical parameters of the graphene sheet are also investigated in order to understand

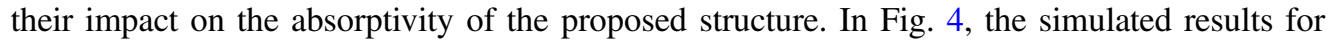
different geometry parameters are reported.

The first observation is the effect of the structure's periodicity " $x$," where by changing the values of $x$ from 515 to $550 \mathrm{~nm}$ with a step width of $5 \mathrm{~nm}$, the absorption rate is found to improve and the ripples start to decline up to a slight ripple obtained at $x=550 \mathrm{~nm}$. The physics behind this is that the dimension of " $x$ " affects both the inter- and intraunits' interactions and subsequently alters the magnetic and electric resonances $\$$ This can also be supported by the LC circuit theory ( $\mathrm{L}$ : inductance and $\mathrm{C}$ : capacitance of the structure), where variation of the parameter " $x$ " affects the capacitance of the structure and for the larger value of " $x$," the magnetic and electric fields tend to be almost overlapped so the ripples become slight 6 四 Another important geometrical parameter is graphene's thickness " $n$." Typically, the thickness of graphene must be small and in this study, it is set to be a fix value $n=1 \mathrm{~nm}$ referring to the one in literature for a graphene-based MTM absorber $\$$ This corresponds roughly to three layers of graphene. By performing simulations of the proposed structure at different thicknesses of graphene sheets, it is realized that the absorptivity of the proposed MTMA unit cell is getting enhanced for smaller values of $n$ and begins to decrease for all thicknesses $>1 \mathrm{~nm}$.

\section{Conclusions}

A graphene-based wideband MTMA was proposed and characterized in this study. Nearly perfect absorption of $99.9 \%$ was achieved at the resonant frequencies and absorption and the wideband response were confirmed by an absorption rate of $>70 \%$, which is gained for the frequency ranging from 537.91 to $635.73 \mathrm{THz}$. A study was conducted on the components of the proposed MTMA unit cell to understand the origin and its wideband absorptivity nature, and it was understood this is a result of strong EM resonances that arise from strong coupling of the structure's resonator with a dielectric spacer and ground metallic plane. As the main objective of this study was to use a graphene sheet for enhancement of the structure absorptivity, the calculated results show that integration of the graphene monolayer on the proposed MTMA unit cell improves its absorption bandwidth up to $26 \%$ of its initial bandwidth without graphene. Finally, the geometry parameters analysis was conducted on the proposed structure after integration of graphene, and it was found that the periodicity of a unit cell structure and the thickness of the graphene layer play a crucial role in their absorptivity (remove or generate ripples on the absorption peaks). The structure proposed here is believed to have some potential applications in the realization of high efficiency solar cells. Experimental study of the proposed MTMA structure remains a subject for future work.

\section{Acknowledgments}

The work reported here was carried out at Middle East Technical University-Northern Cyprus Campus (METU-NCC). It is supported by METU-NCC under the grant numbers of BAP-FEN15-D-3 and BAP-FEN-16-K-8. P. R. acknowledges the financial support of the Government of Rwanda for his graduate studies through the Rwanda Education Board, sponsorship number 911/12.00/SR/2013.

\section{References}

1. V. G. Vesalago and P. N. Labedev, "The electrodynamics of substances with simultaneously negative values of $\varepsilon$ and $\mu$," Sov. Phys. Usp. 10, 509-514 (1968).

2. A. Sihvola, "Metamaterials in electromagnetics," Metamaterials 1, 2-11 (2007).

3. D. R. Smith et al., "Composite medium with simultaneously negative permeability and permittivity," Phys. Rev. Let. 84, 4184-4187 (2000).

4. S. A. Ramakrishna and T. M. Grzegorczyk, Physics and Applications of Negative Refractive Index Materials, CRC Press, Boca Raton, Florida (2008).

5. Y. Q. Huang, J. C. Li, and W. Yang, "Modeling backward wave propagation in metamaterials by finite element time-domain method," SIAM J. Sci. Comput. 35, B248 (2013). 
6. J. B. Chen et al., "Observation of the inverse Doppler effect in negative- index materials at optical frequencies," Nat. Photonics 5, 239-245 (2011).

7. J. B. Pendry, "Negative refraction makes a perfect lens," Phys. Rev. Lett. 85, 3966-3969 (2000).

8. M. W. Feise, P. J. Bevelacqua, and J. B. Schneider, "Effect of surface waves on the behavior of perfect lenses," Phys. Rev. B66, 035113 (2002).

9. Y. Tang et al., "Single-layer metallodielectric nanostructures as dual-band midinfrared filters," Appl. Phys. Lett. 92, 263106 (2008).

10. C. Sabah and S. Uckun, "Multilayer system of Lorentz/Drude type metamaterials with dielectric slabs and its application to electromagnetic filters," Prog. Electromagn. Res. 91, 349-364 (2009).

11. J. Zhu and G. V. Eleftheriades, "Dual-band metamaterial inspired small monopole antenna for WiFi applications," Electron. Lett. 45, 1104 (2009).

12. H. Chen et al., "Controllable left-handed metamaterial and its application to a steerable antenna," Appl. Phys. Lett. 89, 053509 (2006).

13. T. Chen, S. Li, and H. Sun, "Metamaterials application in sensing," Sensors 12, 2742-2765 (2012).

14. R. Melik et al., "Flexible metamaterials for wireless strain sensing," Appl. Phys. Lett. 95, 181105 (2009).

15. N. Liu et al., "Infrared perfect absorber and its application as plasmonic sensor," Nano Lett. 10, 2342-2348 (2010).

16. Z. Jaksic et al., "A consideration of the use of metamaterials for sensing appliactions: field fluctuations and ultimate performance," T. Opt. A: Pure Appl. Opt. 9, S377 (2007).

17. A. Ishimaru, S. Jaruwatanadilok, and Y. Kuga, "Generalized surface plasmon resonance sensors using metamaterials and negative index materials," Prog. Electromagn. Res. 51, 139-152 (2005).

18. A. V. Kabashin et al., "Polasmonic nanorod metamaterials for biosensing," Nat. Mater. 8 , 867-871 (2009).

19. A. Alu and N. Angheta, "Multifrequency optical invisibility cloak with layered plasmonic shells," Phys. Rev. Lett. 100, 113901 (2008).

20. F. Zolla et al., "Electromagnetic analysis of cylindrical invisibility cloaks and the mirage effect," Opt. Lett. 32, 1069 (2007).

21. Y. Lai et al., "A complementary media invisibility cloak that can cloak objects at a distance outside the cloaking shell," Phys. Rev. Lett. 102, 093901 (2009).

22. J. B. Pendry, D. Schurig, and D. R. Smith, "Controlling electromagnetic fields," Science 312, 1780-1782 (2006).

23. M. C. K. Wiltshire, J. B. Pendry, and J. V. Hajnal, "Sub-wavelength imaging at radio frequency," T. Phys.: Condens. Matter 18, L315 (2006).

24. N. I. Landy et al., "A perfect metamaterial absorber," Phys. Rev. Lett. 100, 207402 (2008).

25. C. L. Holloway et al., "An overview of the theory and applications of metasurfaces: the twodimensional equivalents of metamaterials," IEEE Trans. Antennas Propag. 54, 10-35 (2012).

26. C. M. Watts, X. Liu, and W. J. Padilla, "Metamaterial electromagnetic wave absorbers," Adv. Mater. 24, 98-120 (2012).

27. Y. J. Jen et al., "Deposition of $\mathrm{Ta}_{2} \mathrm{O}_{5}$ upon silver nanorods as an ultra-thin light absorber," Thin Solid Films 567, 38-46 (2014).

28. Y. F. C. Chau et al., "Design of crossing metallic metasurface arrays based on high sensitivity of gap enhancement and transmittance shift for plasmonic sensing applications," J. Phys. D: Appl. Phys. 50, 045105 (2017).

29. Y. F. C. Chau et al., "Plasmonic effects arising from a grooved surface of a gold nanorod," J. Phys. D: Appl. Phys. 50, 125302 (2017).

30. P. Rufangura and C. Sabah, "Theoretical and thermal characterization of a wide-band perfect absorber for application in solar cells," Appl. Phys. A 122, 995 (2016).

31. Y. Liu et al., "Study of energy absorption on solar cell using metamaterials," Sol. Energy $\mathbf{8 6}$, 1586-1599 (2012). 
32. C. Wu et al., "Metamaterial-based integrated plasmonic absorber/emitter for solar thermophotovoltaic systems," J. Opt. 14, 024005 (2012).

33. Y. F. C. Chau et al., "Tunable silver-shell dielectric core nano-beads array for thin-film solar cell application," V. Nanopart. Res. 18, 88 (2016).

34. L. Z. Hsieh et al., "Metal nano-particles sizing by thermal annealing for the enhancement of surface plasmon effects in thin-film solar cells application," Opt. Commun. 370, 85-90 (2016).

35. P. W. Gilberd, "The anomalous skin effect and the optical properties of metals," J. Phys. F: Met. Phys. 12, 1845-1860 (1982).

36. B. Wang, T. Koschny, and C. M. Soukoulis, "Wide-angle and polarization-independent chiral metamaterial absorber," Phys. Rev. B 80, 033108 (2009).

37. J. Sun et al., "An extremely broad band metamaterial absorber based on destructive interference," Opt. Exp. 19, 21155 (2011).

38. H. Tao et al., "A metamaterial absorber for the terahertz regime: design, fabrication and characterization," Opt. Exp. 16, 7181 (2008).

39. H. Tao et al., "Highly flexible wide angle of incidence terahertz metamaterial absorber: design, fabrication, and characterization," Phys. Rev. B 78, 241103 (2008).

40. X. Liu et al., "Infrared spatial and frequency selective metamaterial with near-unity absorbance," Phys. Rev. Lett. 104, 207403 (2010).

41. G. Dayal and S. A. Ramakrishna, "Design of highly absorbing metamaterials for infrared frequencies," Opt. Exp. 20, 17503 (2012).

42. P. Pitchappa et al., "Dual band complementary metamaterial absorber in near infrared region," J. Appl. Phys. 115, 193109 (2014).

43. P. Rufangura and C. Sabah, "Dual-band perfect metamaterial absorber for solar cell applications," Vacuum 120, 68-74 (2015).

44. X. Duan et al., "Polarization-insensitive and wide-angle broadband nearly perfect absorber by tunable planar metamaterials in the visible regime," J. Opt. 16, 125107 (2014).

45. L. Mo et al., "Enhanced broadband absorption in gold by plasmonic tapered coaxial holes," Opt. Exp. 22, 32233 (2014).

46. M. Yan, J. Dai, and M. Qiu, "Lithography-free broadband visible light absorber based on a mono-layer of gold nanoparticles," T. Opt. 16, 025002 (2014).

47. B. Mulla and C. Sabah, "Perfect metamaterial absorber design for solar cell applications," Waves Random Complex Medium 25, 382-392 (2015).

48. F. Dincer et al., "Design of polarization and incident angle insensitive dual-band metamaterial absorber based on isotropic resonator," Prog. Electromagn. Res. 144, 123-132 (2014).

49. P. Rufangura and C. Sabah, "Polarization angle insensitive dual-band perfect metamaterial absorber for solar cell applications," Phys. Stat. Sol. C 12, 1241 (2015).

50. F. Dincer et al., "Polarization angle independent perfect metamaterial absorbers for solar cell applications in the microwave, infrared and visible regime," Prog. Electromagn. Res. 144, 93-101 (2014).

51. P. Rufangura and C. Sabah, "Design and characterization of a novel dual-band perfect metamaterial absorber for solar cell applications," J. Alloys Compd. 671, 43-50 (2016).

52. R. Feng et al., "Dual-band infrared perfect absorber based on asymmetric T-shaped plasmonic array," Opt. Express 22, A335 (2014).

53. M. Yan, J. Dai, and M. Qiu, "Lithography-free broadband visible light absorber based on a mono-layer of gold nanoparticles," V. Opt. 16, 025002 (2014).

54. P. Rufangura and C. Sabah, "Wide-band polarization independent perfect metamaterial absorber based on concentric rings topology for solar cells application," T. Alloys Compd. 680, 473-479 (2016).

55. X. Duan et al., "Polarization-insensitive and wide-angle broadband nearly perfect absorber by tunable planar metamaterials in the visible regime," J. Opt. 16, 125107 (2014).

56. P. Rufangura and C. Sabah, "Polarization insensitive tunable metamaterial perfect absorber for solar cells applications," IET Opt. 10(6), 211-216 (2016).

57. X. Shen et al., "Polarization-independent wide-angle triple-band metamaterial absorber," Opt. Express 19, 9401 (2011). 
58. A. Ndryieuski and A. V. Lavrinenko, "Graphene metamaterials based tunable terahertz absorber: effective surface conductivity approach," Opt. Express 21, 9144 (2013).

59. R. Alaee et al., "A perfect absorber made of a graphene micro-ribbon metamaterial," Opt. Express 20, 28017 (2012).

60. K. S. Novoselov et al., "Electric field in atomically thin carbon films," Science 306, 666-669 (2004).

61. W. Zhu, I. D. Rukhlenko, and M. Premaratne, "Graphene metamaterial for optical reflection modulation," Appl. Phys. Lett. 102, 241914 (2013).

62. W. Li et al., "Polarization-insensitive wide-angle multiband metamaterial absorber with a double-layer modified electric ring resonator array," AIP Adv. 5, 067151 (2015).

63. O. Loebich, "The optical properties of gold: a review of their technical utilization," Gold Bult. 5, 2-10 (1972).

64. S. M. Rao, G. K. Gothard, and D. R. Wilton, "Application of finite integration technique to electromagnetic scattering by two-dimensional cavity-backed aperture in a ground plane," IEEE Trans. Antennas Propag. 46, 679-685 (1998).

65. R. Marklein, "The finite integration technique as general tool to compute acoustic, electromagnetic, elastodynamic, and coupled wave fields," EEEE Antennas Wireless Propag. Lett. 1, 31-34 (2002).

66. S. Gutschling, H. Krger, and T. Weiland, "Time domain simulation of dispersive media with the finite integration technique," Int. J. Numer. Modell. 13, 329-348 (2000).

67. G. W. Hanson, "Dyadic greens functions and guided surface waves on graphene," t. Appl. Phys. 103, 064302 (2008).

68. M. Idrio et al., "Graphene assisted critically coupled optical ring modulator," Opt. Express 20, 23144 (2012).

69. A. Vakil and N. Engheta, "Transformation optics using graphene," Science 332, 1291-1294 (2011).

70. J. Wang et al., "Tunable broad-band perfect absorber by exciting of multiple plasmon resonances at optical frequency," Opt. Express 20, 14871-14878 (2012).

Patrick Rufangura: Biography is not available.

Cumali Sabah received his BSc, MSc, and PhD degrees in electrical and electronics engineering. Currently, he is an associate professor of Electrical and Electronics Engineering Department and assistant to the president at Middle East Technical University-Northern Cyprus Campus (METU-NCC). His research interests include the microwave and electromagnetic investigation of unconventional materials and structures, wave propagation, scattering, complex media, metamaterials and their applications, and solar systems. 